\title{
Depressed mood, suicidal behaviors, and health risk behaviors among youths in the Commonwealth of the Northern Mariana Islands: the 2017 CNMI Youth Risk Behavior Survey
}

Jennifer Lisa Sakamoto, Akira Shibanuma* and Masamine Jimba

\begin{abstract}
Background: The current study investigated the prevalence of depressed mood, suicide ideation, suicide plan, and suicide attempt and their associations with health risk behaviors among high school adolescents in the Commonwealth of the Northern Mariana Islands (CNMI).

Methods: This is a cross-sectional study analyzing self-reported data from the 2017 CNMI Youth Risk Behavior Survey ( $n=1943$ ). Modified Poisson regression models were used to identify the associations between 17 health risk behavior variables, including violence-related behaviors, substance use behaviors, sexual behaviors, and early risk-taking behaviors, and four variables related to depressed mood and suicidal behaviors.

Results: $40.7 \%$ adolescents reported being depressed, 25.0\% reported suicide ideation, $22.8 \%$ reported formulating a suicide plan, and $13.6 \%$ attempted suicide. Female adolescents were more likely to report depressed mood and all included suicidal behaviors $(p<0.001)$. Being in a physical fight and forced sexual intercourse were associated with depressed mood, suicide ideation, suicide plan, and suicide attempt for both female and male adolescents. Use of "soft drugs" such as current smoking was associated with depressed $\operatorname{mood}(\mathrm{ARR}=2.33,95 \% \mathrm{Cl}=1.56-3.45, p<0.001)$, suicide ideation ( $\mathrm{ARR}=1.23$, $95 \% \mathrm{Cl}=1.08-1.43, p<0.001)$, suicide plan (ARR $=1.19 ; 95 \% \mathrm{Cl}=1.05-1.35 ; p<0.001)$, and suicide attempt (ARR = 1.18; $95 \% \mathrm{Cl}=1.06-1.30 ; p<0.001)$ for females, whereas use of "hard drugs" such as heroin was associated with depressed mood (ARR $=2.27,95 \% \mathrm{Cl}=1.37-3.85, p<0.01$ ), suicide ideation ( $A R R=1.30,95 \% \mathrm{Cl}=1.01-1.67, p<0.05$ ), suicide plan $(A R R=1.82 ; 95 \% \mathrm{Cl}=1.22-2.70 ; p<0.01)$, and suicide attempt $(\mathrm{ARR}=2.78 ; 95 \% \mathrm{Cl}=1.47-5.26 ; p<0.01)$ for male adolescents.
\end{abstract}

Conclusion: The prevalence of depressed mood, suicide ideation, suicide plan, and suicide attempt among CNMI adolescents was high, especially in female adolescents. While there were gender differences, many of the health risk behaviors were associated with depressed mood and suicidal behaviors. As sociodemographic factors are difficult to change, modifiable factors should be targeted to improve the mental health of adolescents.

Keywords: Depressed mood, Suicidal behavior, Risk behavior, Adolescent, CNMI, Pacific Island

\footnotetext{
* Correspondence: shibanuma@m.u-tokyo.ac.jp

Department of Community and Global Health, Graduate School of Medicine,

The University of Tokyo, Tokyo, Japan
}

C C The Author(s). 2020 Open Access This article is licensed under a Creative Commons Attribution 4.0 International License, which permits use, sharing, adaptation, distribution and reproduction in any medium or format, as long as you give appropriate credit to the original author(s) and the source, provide a link to the Creative Commons licence, and indicate if changes were made. The images or other third party material in this article are included in the article's Creative Commons licence, unless indicated otherwise in a credit line to the material. If material is not included in the article's Creative Commons licence and your intended use is not permitted by statutory regulation or exceeds the permitted use, you will need to obtain permission directly from the copyright holder. To view a copy of this licence, visit http://creativecommons.org/licenses/by/4.0/. The Creative Commons Public Domain Dedication waiver (http://creativecommons.org/publicdomain/zero/1.0/) applies to the data made available in this article, unless otherwise stated in a credit line to the data. 


\section{Background}

Suicide among adolescents is a major public health concern worldwide. In the US, suicide is the second leading cause of death among adolescents aged 10 to 24 years [1]. Approximately 6,000 adolescents died by suicide in 2017, and many more seriously considered and attempted suicide [2]. Those who experience a suicidal crisis typically engage in a continuum of suicidal behaviors from seriously considering suicide (suicide ideation), formulating plans of suicide (suicide plan), and carrying out suicidal acts (suicide attempt) to death by suicide [3-5]. Many people who have ever considered or attempted suicide did so for the first time during their youth, as the lifetime age of onset for suicidal ideation and suicide attempt typically occurs during adolescence to early adulthood [6]. Moreover, depressive symptoms are strongly associated with suicidal behaviors, and experiencing depression during adolescence can lead to severe adverse outcomes later in adulthood [3, 5, 7].

Suicide deaths among adolescents are preventable by overcoming risk factors such as bullying [7], victimization [7], substance abuse [8], and sexual activity [9]. Because suicide is often a complex interaction of psychological, social, biological, cultural, and environmental factors [10, $11]$, it is crucial to identify the risk factors from various aspects of an adolescent's life.

In the Micronesia region encompassing the former US Trust Territories of the Pacific Islands-which include the three inhabited unincorporated island territories of Guam, the Commonwealth of the Northern Mariana Islands (CNMI), and American Samoa-the adolescent suicide rate is among the highest in the world [12, 13]. Specifically, the suicide rate among male adolescents is high in this region, and prior research has explained this epidemiological finding [14]. In the past two centuries, US territories of the Pacific have faced major sociocultural changes through the colonial rule of Spain, Germany, Japan, and finally the US. After World War II until the mid-1960s, rapid cultural changes occurred as westernization greatly influenced society through the introduction of American-style schools and health services and modern forms of technology. Economic changes accompanied these sociocultural changes. The islands shifted from a subsistence economy relying on fishing and farming to a cash economy and widespread government employment [15].

Alongside this major economic transformation, fundamental changes in interpersonal relationships were simultaneously occurring, which led to intergenerational conflict and isolation among adolescents in this region [16]. More recently, a study conducted in Guam suggests that unemployment is particularly high among male adolescents in the US territories of the Pacific because they often lack the skills required for private-sector employment and other high-level jobs. This results in loss of social connections related to employment, resulting in further isolation from society [17]. Although the high suicide rates in the Micronesian region, including the US territories of the Pacific, have drawn attention, most related research has focused primarily on male adolescents and suicide deaths. Previous research has explored suicidal behaviors among US high school adolescents through an analysis of the Youth Risk Behavior Survey (YRBS) [5, 18], a school-based survey conducted by the Centers for Disease Control and Prevention (CDC). Through this survey, the Centers monitor six categories of health-related behaviors-behaviors that lead to unintentional injuries and violence, risky sexual behaviors, alcohol and other drug use, unhealthy dietary behaviors, and inadequate physical activity, all of which contribute to mortality and morbidity among adolescents. However, all US territories, including the CNMI, were excluded from the national YRBS data during the sample selection process [19]. Due to the distinct history and culture of the indigenous population in the US territories of the Pacific [20], the results of the CNMI YRBS might differ from the US national YRBS results.

The current study was conducted to investigate the prevalence of depressed mood and suicidal behaviors and their association with health risk behaviors among high school adolescents in the CNMI.

\section{Methods}

\section{Data source and participants}

This is a cross-sectional study analyzing data from the 2017 CNMI YRBS. The YRBS is sponsored by the CDC and is conducted biennially to monitor health risk behaviors that contribute to the leading causes of death, disability, and social problems among adolescents and adults in the US. The CNMI Public School System conducted the 2017 CNMI YRBS among 9th to 12th graders in CNMI public schools using a two-stage cluster sample design to produce an island-wide representative sample of students. Survey procedures were designed to protect adolescents' privacy through anonymity and encourage voluntary participation. Before administering the survey, local parental permission procedures were followed; these included handing out parental permission forms to the students prior to the survey, and parents who did not want their children to participate in the survey could return the permission forms to the teachers. Adolescents whose parents granted permission to participate completed the self-administered questionnaire on a computer-scannable answer sheet. Because the data used in these analyses contain no personally identifiable information, this study was exempt from institutional board review. Among the three US Pacific territories (Guam, 
Puerto Rico, CNMI) that conducted the YRBS in 2017, CNMI had the largest sample size of 1943 adolescents and an overall response rate of $64 \%$. A weight was applied to each record to adjust for student nonresponse and equitable distribution of students by grade and gender.

In 2017, the national YRBS questionnaire included 99 questions. The 2017 CNMI YRBS included 89 questions from the national YRBS questionnaire that were relevant to its cultural context.

\section{Depressed mood and suicidal behaviors}

The outcome variables were depressed mood and suicidal behaviors, which were measured by four questions in the CNMI YRBS under behaviors related to violence: depressed mood, suicide ideation, suicide plan, and suicide attempt. The test re-test conducted by the CDC to measure the reliability of the four questions showed moderate to substantial reliability [21]. Specifically, kappa values of $56.4 \%$ for depressed mood, $74.3 \%$ for suicide ideation, $66.6 \%$ for suicide plan, and $72.7 \%$ for suicide attempt were obtained [22]. Another study measuring the validity of suicidality items in the YRBS found that these items are valid and thus useful for assessing suicidal behaviors [23]. Depressed mood was assessed with the question: "During the past 12 months, did you ever feel so sad or hopeless almost every day for two weeks or more in a row that you stopped doing some usual activities?" Suicide ideation was assessed with the question: "During the past 12 months, did you ever seriously consider attempting suicide?" Suicide plan was assessed with the question: "During the past 12 months, did you make a plan about how you would attempt suicide?" Suicide attempt was assessed with the question: "During the past 12 months, how many times did you actually attempt suicide?"

\section{Health risk behaviors}

Health risk behaviors were measured by 17 independent variables and classified into four domains: violencerelated behaviors, substance use behaviors, sexual behaviors, and early risk-taking behaviors. The questions and response choices are specified in Table 1.

Violence-related behaviors were assessed with five questions regarding: carrying a weapon, physical fight, forced sexual intercourse, physical dating violence, and being bullied on school property. Substance use behaviors were assessed with six questions regarding: current smoking, current alcohol use, current marijuana use, ever cocaine use, ever heroin use, and ever methamphetamine use. Sexual behaviors were assessed with two questions regarding: current sexual activity status and sex with four or more partners in life. Early risk-taking behaviors were assessed with four questions regarding: smoking under age 13, drinking under age 13, marijuana use under age 13, and sex under age 13.

\section{Demographics}

Demographic information, including age, gender, grade, and race/ethnicity, were all self-reported. Age was reported as one of the following categories: 12 years or younger, 13 years, 14 years, 15 years, 16 years, 17 years, or 18 years or older. Gender was reported as female or male. Grade was reported as one of the following categories: 9th grade, 10th grade, 11th grade, 12th grade, or ungraded/other. Race/ethnicity was reported as one of the following categories: Asian, Native Hawaiian or other Pacific Islander, Black or African American, Hispanic/ Latino, White, American Indian/Alaskan Native, Hispanic with Multiple Race/Ethnicity, or Non-Hispanic Multiple Race/Ethnicity.

Academic performance was self-reported and assessed with the question: "During the past 12 months, how would you describe your grades in school?" The response options were Mostly A's, Mostly B's, Mostly C's, Mostly D's, Mostly F's, and Not sure.

\section{Statistical analyses}

To account for the complex sample design of the YRBS, all analyses used the complex sample survey procedures in software program Stata version 13 (StataCorp, College Station, TX) using svy commands. Prevalence estimates reported herein reflect weighted estimates. Due to the strongly positively skewed distributions, depressed mood, suicidal behavior, and health risk behavior questions were dichotomized as "yes" or "no." The alpha was set at $p<0.05$. A Rao-Scott chi-square test was used to separately assess the association between depressed mood, suicidal behaviors, and demographic variables. Modified Poisson regression models were used to separately assess the association of each health risk behavior variable with each of the following outcomes: depressed mood, suicide ideation, suicide plan, and suicide attempt $[24,25]$. Adjusted relative risks (adjusting for age, race/ ethnicity, and academic performance) along with 95\% confidence intervals were reported. Multicollinearity was tested using tolerance and the variance inflation factor; as a result, grade was excluded but age was retained in all adjusted models.

\section{Results}

Demographic characteristics, academic performance, and health risk behaviors of adolescents

A total of 1943 high school adolescents took the 2017 CNMI YRBS (Table 2). Among these, 49.8\% identified as female adolescents. Most adolescents identified as Native Hawaiian or Pacific Islander (46.5\%), followed by Asian (41.1\%). Academic performance was self-reported, and 
Table 1 Questions on depressed mood, suicidal behaviors, and health risk behaviors with response choices

\begin{tabular}{|c|c|c|}
\hline & Questions & Response choices \\
\hline \multicolumn{3}{|l|}{ Depressed mood } \\
\hline Depressed mood & $\begin{array}{l}\text { During the past } 12 \text { months, did you ever feel so sad or hopeless almost } \\
\text { every day for two weeks or more in a row that you stopped doing some } \\
\text { usual activities? }\end{array}$ & 1: Yes; 2: No \\
\hline \multicolumn{3}{|l|}{ Suicidal behaviors } \\
\hline Suicide ideation & $\begin{array}{l}\text { During the past } 12 \text { months, did you ever seriously consider attempting } \\
\text { suicide? }\end{array}$ & 1: Yes; 2: No \\
\hline Suicide plan & $\begin{array}{l}\text { During the past } 12 \text { months, did you make a plan about how you would } \\
\text { attempt suicide? }\end{array}$ & 1: Yes; 2: No \\
\hline Suicide attempt & $\begin{array}{l}\text { During the past } 12 \text { months, how many times did you actually attempt } \\
\text { suicide? }\end{array}$ & 1: 0 times; $2: 1+$ time \\
\hline \multicolumn{3}{|l|}{ Violence } \\
\hline Carried a weapon & $\begin{array}{l}\text { During the past } 30 \text { days, on how many days did you carry a weapon such as } \\
\text { gun, knife, or club? }\end{array}$ & 1: 0 days; $2: 1+$ day \\
\hline Physical fight & During the past 12 months, how many times were you in a physical fight? & 1: 0 times; $2: 1+$ time \\
\hline $\begin{array}{l}\text { Forced sexual } \\
\text { intercourse }\end{array}$ & $\begin{array}{l}\text { Have you ever been physically forced to have sexual intercourse when you } \\
\text { did not want to? }\end{array}$ & 1: Yes; 2: No \\
\hline $\begin{array}{l}\text { Physical dating } \\
\text { violence }\end{array}$ & $\begin{array}{l}\text { During the past } 12 \text { months, how many times did someone you were dating } \\
\text { or going out with physically hurt you on purpose? }\end{array}$ & $\begin{array}{l}\text { 1: I did not date or go out with anyone during } \\
\text { the past } 12 \text { months or } 0 \text { times; } 2: 1+\text { time }\end{array}$ \\
\hline $\begin{array}{l}\text { Bullied on school } \\
\text { property }\end{array}$ & During the past 12 months, have you ever been bullied on school property? & 1: Yes; 2: No \\
\hline \multicolumn{3}{|l|}{ Substance use } \\
\hline Current smoking & During the past 30 days, on how many days did you smoke cigarettes? & 1: 0 days; $2: 1+$ day \\
\hline Current alcohol use & $\begin{array}{l}\text { During the past } 30 \text { days, on how many days did you have at least one drink } \\
\text { of alcohol? }\end{array}$ & 1: 0 days; $2: 1+$ day \\
\hline $\begin{array}{l}\text { Current marijuana } \\
\text { use }\end{array}$ & $\begin{array}{l}\text { During the past } 30 \text { days, how many times did you use marijuana on school } \\
\text { property? }\end{array}$ & 1: 0 times; $2: 1+$ time \\
\hline Ever cocaine use & $\begin{array}{l}\text { During your life, how many times have you used any form of cocaine, } \\
\text { including powder, crack, or freebase? }\end{array}$ & 1: 0 times; $2: 1+$ time \\
\hline Ever heroin use & $\begin{array}{l}\text { During your life, how many times have you used heroin (also called smack, } \\
\text { junk, or China White)? }\end{array}$ & 1: 0 times; $2: 1+$ time \\
\hline $\begin{array}{l}\text { Ever } \\
\text { methamphetamine } \\
\text { use }\end{array}$ & $\begin{array}{l}\text { During your life, how many times have you used methamphetamines (also } \\
\text { called speed, crystal, crank, or ice)? }\end{array}$ & 1: 0 times; $2: 1+$ time \\
\hline \multicolumn{3}{|l|}{ Sexual behavior } \\
\hline $\begin{array}{l}\text { Currently sexually } \\
\text { active }\end{array}$ & $\begin{array}{l}\text { During the past } 3 \text { months, how many people have you had sexual } \\
\text { intercourse with? }\end{array}$ & $\begin{array}{l}1: \text { I did not date or go out with anyone during } \\
\text { the past } 12 \text { months or } 0 \text { person; } 2: 1+\text { people }\end{array}$ \\
\hline $\begin{array}{l}\text { Sex with } 4 \text { or more } \\
\text { in life }\end{array}$ & During your life, with how many people have you had sexual intercourse? & $\begin{array}{l}\text { 1: I have never had sexual intercourse or } 1-3 \\
\text { people; } 2: 4+\text { people }\end{array}$ \\
\hline \multicolumn{3}{|c|}{ Early risk-taking behavior } \\
\hline $\begin{array}{l}\text { Smoking under age } \\
13\end{array}$ & How old were you when you smoked a whole cigarette for the first time? & $\begin{array}{l}\text { 1: I have never smoked or } 13 \text { years old }+; 2: 12 \\
\text { years old or younger }\end{array}$ \\
\hline $\begin{array}{l}\text { Drinking under age } \\
13\end{array}$ & How old were you when you had your first alcohol other than a few sips? & $\begin{array}{l}1: \text { I have never had a drink or } 13 \text { years old }+; 2 \text { : } \\
12 \text { years old or younger }\end{array}$ \\
\hline $\begin{array}{l}\text { Marijuana use } \\
\text { under age } 13\end{array}$ & How old were you when you tried marijuana for the first time? & $\begin{array}{l}\text { 1: I have never tried marijuana or } 13 \text { years oldt; } \\
\text { 2: } 12 \text { years old or younger }\end{array}$ \\
\hline Sex under age 13 & How old were you when you had sexual intercourse for the first time? & $\begin{array}{l}\text { 1: I have never had sexual intercourse or } 13 \text { years } \\
\text { old +; } 2 \text { : } 12 \text { years old or younger }\end{array}$ \\
\hline
\end{tabular}


Table 2 Demographic variables and academic performance of adolescents

\begin{tabular}{|c|c|c|}
\hline & \multicolumn{2}{|c|}{ Total $(\boldsymbol{n}=1943)$} \\
\hline & $\begin{array}{l}\text { Unweighted } \\
\text { Frequency (n) }\end{array}$ & $\begin{array}{l}\text { Weighted } \\
\text { Frequency (\%) }\end{array}$ \\
\hline \multicolumn{3}{|l|}{ Demographic variables } \\
\hline \multicolumn{3}{|l|}{ Age } \\
\hline$<=14$ years old & 207 & 9.6 \\
\hline 15 years old & 536 & 25.2 \\
\hline 16 years old & 547 & 28.5 \\
\hline 17 years old & 453 & 25.7 \\
\hline$>=18$ years old & 199 & 11.0 \\
\hline Missing & 1 & \\
\hline \multicolumn{3}{|l|}{ Gender } \\
\hline Female & 951 & 49.8 \\
\hline Male & 982 & 50.2 \\
\hline Missing & 10 & \\
\hline \multicolumn{3}{|l|}{ Grade } \\
\hline 9th grade & 623 & 29.3 \\
\hline 10th grade & 557 & 26.8 \\
\hline 11th grade & 502 & 31.1 \\
\hline 12th grade & 242 & 12.7 \\
\hline Ungraded or other grade & 5 & 0.3 \\
\hline Missing & 14 & \\
\hline \multicolumn{3}{|l|}{ Race/Ethnicity } \\
\hline Asian & 762 & 41.1 \\
\hline Pacific Islander & 874 & 46.5 \\
\hline Black & 11 & 0.6 \\
\hline Hispanic & 2 & 0.1 \\
\hline White & 12 & 0.7 \\
\hline $\begin{array}{l}\text { American Indian/Alaskan } \\
\text { Native }\end{array}$ & 2 & 0.1 \\
\hline Multiple-Hispanic/Latino & 103 & 5.6 \\
\hline $\begin{array}{l}\text { Multiple-Non Hispanic/ } \\
\text { Latino }\end{array}$ & 100 & 5.3 \\
\hline Missing & 77 & \\
\hline \multicolumn{3}{|l|}{ Academic performance } \\
\hline Mostly A's & 604 & 33.0 \\
\hline Mostly B's & 593 & 32.2 \\
\hline Mostly C's & 314 & 17.2 \\
\hline Mostly D's & 125 & 6.7 \\
\hline Mostly F's & 31 & 1.4 \\
\hline None of these grades & 5 & 0.3 \\
\hline Not sure & 174 & 9.3 \\
\hline Missing & 97 & \\
\hline
\end{tabular}

most reported receiving A's (33.1\%) or B's (32.1\%). Because of the small sample size, adolescents aged 12 years or younger, 13 years, and 14 years were combined into one category.

Table 3 shows the prevalence of health risk behaviors among the adolescents in unweighted frequencies and weighted percentages. Among the adolescents, 502 (26.0\%) reported using marijuana at least one or more times in the past 30 days and 393 (24.3\%) reported being currently sexually active.

Prevalence of depressed mood and suicidal behaviors

Overall, $786(40.7 \%)$ adolescents reported that they were in a depressed mood every day for two or more weeks in a row, causing them to stop doing some usual activities during the 12 months before the survey; in addition, 498 (25.0\%) adolescents reported suicide ideation, 459 (22.8\%) reported formulating a suicide plan, and 241 (13.6\%) attempted suicide during the past 12 months. As Table 4 shows, female adolescents were significantly more likely to report depressed mood and all included suicidal behaviors than male adolescents. Differences by

Table 3 Prevalence of health risk behaviors among adolescents

\begin{tabular}{|c|c|c|}
\hline \multirow[b]{2}{*}{ Health risk behaviors } & \multicolumn{2}{|l|}{ Total $(\boldsymbol{n}=1943)$} \\
\hline & $\begin{array}{l}\text { Unweighted } \\
\text { Frequency (n) }\end{array}$ & $\begin{array}{l}\text { Weighted } \\
\text { Frequency (\%) }\end{array}$ \\
\hline \multicolumn{3}{|l|}{ Violence-related behaviors } \\
\hline Carried a weapon & 264 & 13.0 \\
\hline Physical fight & 410 & 20.9 \\
\hline Forced sexual intercourse & 245 & 13.1 \\
\hline Physical dating violence & 94 & 9.1 \\
\hline $\begin{array}{l}\text { Bullied on school } \\
\text { property }\end{array}$ & 464 & 23.2 \\
\hline \multicolumn{3}{|l|}{ Substance use behaviors } \\
\hline Current smoking & 231 & 12.4 \\
\hline Current alcohol use & 381 & 23.3 \\
\hline Current marijuana use & 502 & 26.0 \\
\hline Ever use of cocaine & 70 & 3.6 \\
\hline Ever use of heroin & 56 & 3.0 \\
\hline $\begin{array}{l}\text { Ever use of } \\
\text { methamphetamine }\end{array}$ & 59 & 3.4 \\
\hline \multicolumn{3}{|l|}{ Sexual behaviors } \\
\hline Currently sexually active & 393 & 24.3 \\
\hline Sex with 4 or more in life & 99 & 5.9 \\
\hline \multicolumn{3}{|c|}{ Early risk-taking behaviors } \\
\hline Smoking under age 13 & 328 & 17.4 \\
\hline Drinking under age 13 & 336 & 18.7 \\
\hline $\begin{array}{l}\text { Marijuana use under age } \\
13\end{array}$ & 239 & 12.5 \\
\hline Sex under age 13 & 69 & 4.1 \\
\hline
\end{tabular}


Table 4 Prevalence of depressed mood and suicidal behaviors by demographic variables and academic performance

\begin{tabular}{|c|c|c|c|c|c|c|c|c|}
\hline \multirow[t]{2}{*}{ Demographic variables } & \multicolumn{2}{|c|}{ Depressed mood } & \multicolumn{2}{|c|}{ Suicide ideation } & \multicolumn{2}{|l|}{ Suicide plan } & \multicolumn{2}{|c|}{ Suicide attempt } \\
\hline & Yes $(\boldsymbol{n}=786)$ & $\boldsymbol{p}$-value & Yes $(\boldsymbol{n}=498)$ & $\boldsymbol{p}$-value & Yes $(\boldsymbol{n}=459)$ & $\boldsymbol{p}$-value & Yes $(\boldsymbol{n}=241)$ & $\boldsymbol{p}$-value \\
\hline Gender & & $<0.0001$ & & $<0.0001$ & & $<0.0001$ & & $<0.0001$ \\
\hline Female & 49.5 & & 32.5 & & 28.4 & & 17.2 & \\
\hline Male & 32.0 & & 17.5 & & 17.2 & & 9.8 & \\
\hline Age & & 0.211 & & 0.286 & & 0.325 & & 0.047 \\
\hline$<=14$ years old & 9.4 & & 8.8 & & 10.7 & & 10.9 & \\
\hline 15 years old & 26.8 & & 25.6 & & 26.3 & & 28.4 & \\
\hline 16 years old & 30.1 & & 31.6 & & 31.5 & & 31.7 & \\
\hline 17 years old & 24.6 & & 22.7 & & 23.3 & & 21.5 & \\
\hline$>=18$ years old & 9.1 & & 11.3 & & 8.3 & & 7.5 & \\
\hline Grade & & 0.062 & & 0.809 & & 0.536 & & 0.446 \\
\hline 9th grade & 45.0 & & 25.5 & & 25.0 & & 15.0 & \\
\hline 10th grade & 40.2 & & 23.9 & & 23.7 & & 14.4 & \\
\hline 11th grade & 40.5 & & 24.6 & & 21.3 & & 12.7 & \\
\hline 12th grade & 33.0 & & 27.0 & & 19.4 & & 8.8 & \\
\hline Race/Ethnicity & & 0.137 & & 0.041 & & 0.474 & & 0.045 \\
\hline Asian & 39.6 & & 21.8 & & 20.9 & & 10.0 & \\
\hline Pacific Islander & 41.3 & & 28.5 & & 24.9 & & 15.5 & \\
\hline Black & 21.3 & & 33.2 & & 19.9 & & 16.6 & \\
\hline Hispanic & 44.2 & & 0.0 & & 0.0 & & 0.0 & \\
\hline White & 11.5 & & 0.0 & & 16.2 & & 12.4 & \\
\hline Academic performance & & 0.042 & & 0.001 & & 0.002 & & 0.001 \\
\hline Mostly A's & 35.0 & & 22.3 & & 19.3 & & 10.3 & \\
\hline Mostly B's & 41.8 & & 21.7 & & 21.8 & & 11.2 & \\
\hline Mostly C's & 41.1 & & 27.7 & & 23.2 & & 14.9 & \\
\hline Mostly D's & 47.9 & & 27.1 & & 25.7 & & 16.1 & \\
\hline Mostly F's & 58.8 & & 57.8 & & 54.9 & & 32.5 & \\
\hline
\end{tabular}

Note. Rao-Scott chi-square test was used to test the independence regarding the combination of a demographic or academic performance variable and an outcome

grade were not observed in depressed mood and suicidal behaviors (Table 4).

\section{Violence-related behaviors associated with depressed mood and suicidal behaviors}

Table 5 and Table 6 show the adjusted relative risk (ARR) and unadjusted relative risk (RR) of health risk behaviors associated with depressed mood and suicidal behaviors. Being involved in a physical fight and forced sexual intercourse were positively associated with depressed mood, suicide ideation, suicide plan, and suicide attempt for both male and female adolescents. Carrying a weapon was associated with suicide ideation $(A R R=$ 1.12 , 95\% confidence interval $(\mathrm{CI})=1.01-1.23, p<0.05)$ and suicide attempt $(\mathrm{ARR}=1.10,95 \% \mathrm{CI}=1.01-1.20$, $p<0.05$ ) only for males. Experiencing bullying on school property was positively associated with depressed mood $(\mathrm{ARR}=1.59,95 \% \mathrm{CI}=1.28-1.96, p<0.001)$, suicide ideation $(\mathrm{ARR}=1.39,95 \% \mathrm{CI}=1.19-1.61, p<0.001)$, suicide plan (ARR $=1.33,95 \% \mathrm{CI}=1.16-1.52, p<0.001)$, and suicide attempt $(\mathrm{ARR}=1.27,95 \% \mathrm{CI}=1.14-1.39, p<$ 0.001 ) for female adolescents.

Substance use behaviors associated with depressed mood and suicidal behaviors

Being a current smoker, current alcohol user, and current marijuana user were associated with depressed mood, suicide ideation, suicide plan, and suicide attempt exclusively for female adolescents. Among male adolescents, ever use of heroin (depressed mood: $A R R=2.17$, 95\% $\mathrm{CI}=1.35-3.45, p<0.01$; suicide plan: $\mathrm{ARR}=1.85$, 95\% $\mathrm{CI}=1.20-2.86, \quad p<0.01$; suicide attempt: $\mathrm{ARR}=$ 
Table 5 Association between depressed mood, suicidal behaviors, and health risk behaviors of adolescents by gender, adjusted relative risk

\begin{tabular}{|c|c|c|c|c|c|c|c|c|}
\hline \multirow[b]{2}{*}{ Health risk behaviors } & \multicolumn{2}{|c|}{ Depressed mood } & \multicolumn{2}{|c|}{ Suicide ideation } & \multicolumn{2}{|l|}{ Suicide plan } & \multicolumn{2}{|c|}{ Suicide attempt } \\
\hline & $\begin{array}{l}\text { Female } \\
(n=472), \text { ARR } \\
(95 \% \mathrm{Cl})\end{array}$ & $\begin{array}{l}\text { Male } \\
(n=332), \text { ARR } \\
(95 \% \mathrm{Cl})\end{array}$ & $\begin{array}{l}\text { Female } \\
(n=347), \text { ARR } \\
(95 \% \mathrm{Cl})\end{array}$ & $\begin{array}{l}\text { Male } \\
(n=195), \text { ARR } \\
(95 \% \text { Cl) }\end{array}$ & $\begin{array}{l}\text { Female } \\
(n=336), \text { ARR } \\
(95 \% \mathrm{Cl})\end{array}$ & $\begin{array}{l}\text { Male } \\
(n=218), \text { ARR } \\
(95 \% \mathrm{Cl})\end{array}$ & $\begin{array}{l}\text { Female } \\
(n=193), \text { ARR } \\
(95 \% \mathrm{Cl})\end{array}$ & $\begin{array}{l}\text { Male } \\
(n=92), \text { ARR } \\
(95 \% \mathrm{Cl})\end{array}$ \\
\hline \multicolumn{9}{|l|}{ Violence } \\
\hline Carried a weapon & $\begin{array}{l}\text { 1. } 49(0.99 \\
2.22)\end{array}$ & $\begin{array}{l}1.08(0.93, \\
1.23)\end{array}$ & $\begin{array}{l}1.19 \text { (0.94, } \\
1.49)\end{array}$ & $\begin{array}{l}1.12(1.01, \\
1.23)\end{array}$ & $\begin{array}{l}1.18(0.96 \\
1.45)\end{array}$ & $\begin{array}{l}1.10(0.99, \\
1.20)\end{array}$ & $\begin{array}{l}1.18(0.98 \\
1.41)\end{array}$ & $\begin{array}{l}1.10(1.01, \\
1.20)\end{array}$ \\
\hline Physical fight & $\begin{array}{l}1.56(1.18, \\
2.08)\end{array}$ & $\begin{array}{l}1.41(1.20, \\
1.64)\end{array}$ & $\begin{array}{l}1.32(1.08, \\
1.61)\end{array}$ & $\begin{array}{l}1.12(1.03, \\
1.23)\end{array}$ & $\begin{array}{l}1.33(1.15, \\
1.56)\end{array}$ & $\begin{array}{l}1.20(1.08, \\
1.33)\end{array}$ & $\begin{array}{l}1.54(1.28, \\
1.89)\end{array}$ & $\begin{array}{l}1.10(1.03, \\
1.18)\end{array}$ \\
\hline $\begin{array}{l}\text { Forced sexual } \\
\text { intercourse }\end{array}$ & $\begin{array}{l}2.27(1.54, \\
3.33)\end{array}$ & $\begin{array}{l}1.52(1.15 \\
2.04)\end{array}$ & $\begin{array}{l}1.54(1.23 \\
1.92)\end{array}$ & $\begin{array}{l}1.37(1.12, \\
1.67)\end{array}$ & $\begin{array}{l}1.32(1.12, \\
1.54)\end{array}$ & $\begin{array}{l}1.33(1.10 \\
1.61)\end{array}$ & $\begin{array}{l}1.28(1.12, \\
1.47)\end{array}$ & $\begin{array}{l}1.22(1.04, \\
1.43)\end{array}$ \\
\hline Physical dating violence & $\begin{array}{l}1.64(0.88 \\
3.03)\end{array}$ & $\begin{array}{l}1.56(1.12, \\
2.13)\end{array}$ & $\begin{array}{l}1.59(1.04 \\
2.44)\end{array}$ & $\begin{array}{l}1.79(1.37 \\
2.38)\end{array}$ & $\begin{array}{l}1.64(1.15 \\
2.33)\end{array}$ & $\begin{array}{l}1.89(1.33 \\
2.63)\end{array}$ & $\begin{array}{l}1.75(1.22, \\
2.50)\end{array}$ & $\begin{array}{l}1.92(1.33, \\
2.78)\end{array}$ \\
\hline $\begin{array}{l}\text { Bullied on school } \\
\text { property }\end{array}$ & $\begin{array}{l}1.59(1.28, \\
1.96)\end{array}$ & $\begin{array}{l}1.49(1.27 \\
1.82)\end{array}$ & $\begin{array}{l}1.39 \text { (1.19, } \\
1.61)\end{array}$ & $\begin{array}{l}1.23(1.09 \\
1.37)\end{array}$ & $\begin{array}{l}1.33(1.16, \\
1.52)\end{array}$ & $\begin{array}{l}1.33(1.19 \\
1.52)\end{array}$ & $\begin{array}{l}1.27(1.14, \\
1.39)\end{array}$ & $\begin{array}{l}1.06(1.00 \\
1.14)\end{array}$ \\
\hline \multicolumn{9}{|l|}{ Substance use } \\
\hline Current smoking & $\begin{array}{l}2.33(1.56, \\
3.45)\end{array}$ & $\begin{array}{l}1.08(0.93 \\
1.23)\end{array}$ & $\begin{array}{l}1.64(1.27 \\
2.13)\end{array}$ & $\begin{array}{l}1.05(0.94 \\
1.18)\end{array}$ & $\begin{array}{l}1.45 \text { (1.16, } \\
1.79)\end{array}$ & $\begin{array}{l}1.06(0.97 \\
1.16)\end{array}$ & $\begin{array}{l}1.59(1.25 \\
2.04)\end{array}$ & $\begin{array}{l}1.11(1.01, \\
1.20)\end{array}$ \\
\hline Current alcohol use & $\begin{array}{l}1.54(1.22, \\
1.96)\end{array}$ & $\begin{array}{l}1.15(1.00, \\
1.33)\end{array}$ & $\begin{array}{l}1.28(1.11, \\
1.47)\end{array}$ & $\begin{array}{l}1.06(0.96, \\
1.18)\end{array}$ & $\begin{array}{l}1.27(1.09, \\
1.45)\end{array}$ & $\begin{array}{l}1.14(1.02, \\
1.27)\end{array}$ & $\begin{array}{l}1.23(1.08, \\
1.41)\end{array}$ & $\begin{array}{l}1.08(1.00, \\
1.15)\end{array}$ \\
\hline Current marijuana use & $\begin{array}{l}1.45(1.18, \\
1.79)\end{array}$ & $\begin{array}{l}1.06(0.94 \\
1.22)\end{array}$ & $\begin{array}{l}1.23(1.06, \\
1.43)\end{array}$ & $\begin{array}{l}1.04(0.96, \\
1.14)\end{array}$ & $\begin{array}{l}1.16(1.03, \\
1.33)\end{array}$ & $\begin{array}{l}1.01(0.93, \\
1.09)\end{array}$ & $\begin{array}{l}1.12(1.01, \\
1.25)\end{array}$ & $\begin{array}{l}1.03(0.97 \\
1.10)\end{array}$ \\
\hline Ever cocaine use & $\begin{array}{l}0.72(0.43 \\
1.19)\end{array}$ & $\begin{array}{l}1.41(1.08, \\
1.82)\end{array}$ & $\begin{array}{l}0.80(0.54 \\
1.19)\end{array}$ & $\begin{array}{l}1.19(0.93 \\
1.52)\end{array}$ & $\begin{array}{l}0.88(0.59, \\
1.30)\end{array}$ & $\begin{array}{l}1.30(1.00, \\
1.69)\end{array}$ & $\begin{array}{l}1.61(0.68, \\
3.85)\end{array}$ & $\begin{array}{l}1.64(1.16, \\
2.33)\end{array}$ \\
\hline Ever heroin use & $\begin{array}{l}0.75(0.36 \\
1.56)\end{array}$ & $\begin{array}{l}2.17(1.35, \\
3.45)\end{array}$ & $\begin{array}{l}1.18(0.57 \\
2.44)\end{array}$ & $\begin{array}{l}1.27(0.96 \\
1.67)\end{array}$ & $\begin{array}{l}1.72(0.67, \\
4.55)\end{array}$ & $\begin{array}{l}1.85(1.20 \\
2.86)\end{array}$ & $\begin{array}{l}2.33(0.63 \\
9.09)\end{array}$ & $\begin{array}{l}2.70(1.32, \\
5.56)\end{array}$ \\
\hline $\begin{array}{l}\text { Ever methamphetamine } \\
\text { use }\end{array}$ & $\begin{array}{l}0.73(0.44 \\
1.20)\end{array}$ & $\begin{array}{l}1.72(1.19, \\
2.50)\end{array}$ & $\begin{array}{l}1.18(0.63 \\
2.17)\end{array}$ & $\begin{array}{l}1.16(0.92 \\
1.47)\end{array}$ & $\begin{array}{l}1.39(0.74 \\
2.56)\end{array}$ & $\begin{array}{l}1.64(1.18, \\
2.27)\end{array}$ & $\begin{array}{l}2.13(0.72 \\
6.25)\end{array}$ & $\begin{array}{l}1.79(1.33 \\
2.38)\end{array}$ \\
\hline \multicolumn{9}{|l|}{ Sexual behavior } \\
\hline Currently sexually active & $\begin{array}{l}1.18(0.95 \\
1.47)\end{array}$ & $\begin{array}{l}1.01(0.89, \\
1.15)\end{array}$ & $\begin{array}{l}1.23(1.08, \\
1.43)\end{array}$ & $\begin{array}{l}1.10(0.99 \\
1.20)\end{array}$ & $\begin{array}{l}1.19(1.05, \\
1.35)\end{array}$ & $\begin{array}{l}1.05(0.97 \\
1.15)\end{array}$ & $\begin{array}{l}1.18(1.06, \\
1.30)\end{array}$ & $\begin{array}{l}1.08(1.00 \\
1.16)\end{array}$ \\
\hline $\begin{array}{l}\text { Sex with } 4 \text { or more in } \\
\text { life }\end{array}$ & $\begin{array}{l}1.69(0.93 \\
3.13)\end{array}$ & $\begin{array}{l}1.16(0.93 \\
1.47)\end{array}$ & $\begin{array}{l}1.11(0.83 \\
1.49)\end{array}$ & $\begin{array}{l}1.10(0.89 \\
1.35)\end{array}$ & $\begin{array}{l}1.01(0.81 \\
1.28)\end{array}$ & $\begin{array}{l}1.06(0.92 \\
1.22)\end{array}$ & $\begin{array}{l}1.16(0.93 \\
1.45)\end{array}$ & $\begin{array}{l}1.06(0.97 \\
1.18)\end{array}$ \\
\hline \multicolumn{9}{|l|}{ Early risk-taking behavior } \\
\hline Smoking under age 13 & $\begin{array}{l}1.12(0.93 \\
1.35)\end{array}$ & $\begin{array}{l}1.08(0.95 \\
1.22)\end{array}$ & $\begin{array}{l}1.18(0.99 \\
1.41)\end{array}$ & $\begin{array}{l}1.05(0.96 \\
1.16)\end{array}$ & $\begin{array}{l}1.19(1.02, \\
1.37)\end{array}$ & $\begin{array}{l}1.14(1.03, \\
1.25)\end{array}$ & $\begin{array}{l}1.08(0.95 \\
1.22)\end{array}$ & $\begin{array}{l}1.02(0.95 \\
1.09)\end{array}$ \\
\hline Drinking under age 13 & $\begin{array}{l}1.25(0.98 \\
1.61)\end{array}$ & $\begin{array}{l}1.09(0.94 \\
1.25)\end{array}$ & $\begin{array}{l}1.19(1.04, \\
1.39)\end{array}$ & $\begin{array}{l}1.09(0.98, \\
1.22)\end{array}$ & $\begin{array}{l}1.23(1.08, \\
1.43)\end{array}$ & $\begin{array}{l}1.14(1.02, \\
1.27)\end{array}$ & $\begin{array}{l}1.10(0.99 \\
1.22)\end{array}$ & $\begin{array}{l}1.11(1.03, \\
1.20)\end{array}$ \\
\hline $\begin{array}{l}\text { Marijuana use under age } \\
13\end{array}$ & $\begin{array}{l}0.99(0.75 \\
1.32)\end{array}$ & $\begin{array}{l}1.16(1.00, \\
1.37)\end{array}$ & $\begin{array}{l}1.11(0.88, \\
1.39)\end{array}$ & $\begin{array}{l}1.00(0.89 \\
1.11)\end{array}$ & $\begin{array}{l}1.09(0.92 \\
1.28)\end{array}$ & $\begin{array}{l}1.01(0.91 \\
1.11)\end{array}$ & $\begin{array}{l}1.19(1.00 \\
1.41)\end{array}$ & $\begin{array}{l}1.02(0.95 \\
1.10)\end{array}$ \\
\hline Sex under age 13 & $\begin{array}{l}1.37(0.83 \\
2.22)\end{array}$ & $\begin{array}{l}1.08(0.84 \\
1.37)\end{array}$ & $\begin{array}{l}1.19(0.83 \\
1.69)\end{array}$ & $\begin{array}{l}0.98(0.85 \\
1.12)\end{array}$ & $\begin{array}{l}1.09(0.82 \\
1.43)\end{array}$ & $\begin{array}{l}0.97(0.85 \\
1.11)\end{array}$ & $\begin{array}{l}1.01 \\
1.22)\end{array}$ & $\begin{array}{l}1.00(0.91 \\
1.11)\end{array}$ \\
\hline
\end{tabular}

Note. ARR adjusted relative risk, $\mathrm{Cl}$ confidence interval. Each ARR and its $95 \% \mathrm{Cl}$ was estimated in a separate model, adjusted for age, race/ethnicity, and academic performance. The numbers in bold indicate significance at $p<0.05$

2.70, $95 \% \mathrm{CI}=1.32-5.56, \quad p<0.01)$ and methamphetamine (depressed mood: $\mathrm{ARR}=1.72,95 \% \mathrm{CI}=1.19-2.50$, $p<0.01$; suicide plan: $\mathrm{ARR}=1.64,95 \% \mathrm{CI}=1.18-2.27$, $p<0.01$; suicide attempt: $\mathrm{ARR}=1.79,95 \% \mathrm{CI}=1.33-2.38$, $p<0.001)$ were associated with depressed mood, suicide plan, and suicide attempt, while none of these were associated among females.
Sexual behaviors associated with depressed mood and suicidal behaviors

Regarding sexual behaviors, being currently sexually active was associated with suicide ideation $(\mathrm{ARR}=1.23,95 \% \mathrm{CI}=$ $1.08-1.43, p<0.01)$, suicide plan (ARR $=1.19,95 \% \mathrm{CI}=$ $1.05-1.35, p<0.01$ ), and suicide attempt (ARR $=1.18,95 \%$ $\mathrm{CI}=1.06-1.30, p<0.01)$ for female adolescents. 
Table 6 Association between depressed mood, suicidal behaviors, and health risk behaviors of adolescents by gender, unadjusted relative risk

\begin{tabular}{|c|c|c|c|c|c|c|c|c|}
\hline \multirow[b]{2}{*}{ Health risk behaviors } & \multicolumn{2}{|c|}{ Depressed mood } & \multicolumn{2}{|c|}{ Suicide ideation } & \multicolumn{2}{|l|}{ Suicide plan } & \multicolumn{2}{|c|}{ Suicide attempt } \\
\hline & $\begin{array}{l}\text { Female } \\
(n=472), R R \\
(95 \% \mathrm{Cl})\end{array}$ & $\begin{array}{l}\text { Male } \\
(n=332), R R \\
(95 \% \mathrm{Cl})\end{array}$ & $\begin{array}{l}\text { Female } \\
(n=347), R R \\
(95 \% \mathrm{Cl})\end{array}$ & $\begin{array}{l}\text { Male } \\
(n=195), R R \\
(95 \% \mathrm{Cl})\end{array}$ & $\begin{array}{l}\text { Female } \\
(n=336), R R \\
(95 \% \mathrm{Cl})\end{array}$ & $\begin{array}{l}\text { Male } \\
(n=218), R R \\
(95 \% \mathrm{Cl})\end{array}$ & $\begin{array}{l}\text { Female } \\
(n=193), \text { RR } \\
(95 \% \mathrm{Cl})\end{array}$ & $\begin{array}{l}\text { Male } \\
(n=92), \text { RR } \\
(95 \% \mathrm{Cl})\end{array}$ \\
\hline \multicolumn{9}{|l|}{ Violence } \\
\hline Carried a weapon & $\begin{array}{l}1.49(0.99, \\
2.22)\end{array}$ & $\begin{array}{l}1.15(0.90 \\
1.45)\end{array}$ & $\begin{array}{l}1.32(0.88, \\
1.96)\end{array}$ & $\begin{array}{l}1.28(1.03, \\
1.61)\end{array}$ & $\begin{array}{l}1.45(1.02, \\
2.04)\end{array}$ & $\begin{array}{l}1.11(0.93, \\
1.30)\end{array}$ & $\begin{array}{l}1.47(0.88, \\
2.44)\end{array}$ & $\begin{array}{l}1.25(1.00, \\
1.56)\end{array}$ \\
\hline Physical fight & $\begin{array}{l}1.72(1.32, \\
2.22)\end{array}$ & $\begin{array}{l}1.35(1.18, \\
1.56)\end{array}$ & $\begin{array}{l}1.43(1.15, \\
1.79)\end{array}$ & $\begin{array}{l}1.14(1.05, \\
1.23)\end{array}$ & $\begin{array}{l}1.45(1.22, \\
1.75)\end{array}$ & $\begin{array}{l}1.22(1.11, \\
1.33)\end{array}$ & $\begin{array}{l}1.61(1.35, \\
1.96)\end{array}$ & $\begin{array}{l}1.14(1.05, \\
1.22)\end{array}$ \\
\hline $\begin{array}{l}\text { Forced sexual } \\
\text { intercourse }\end{array}$ & $\begin{array}{l}2.33(1.67, \\
3.23)\end{array}$ & $\begin{array}{l}1.45(1.14, \\
1.85)\end{array}$ & $\begin{array}{l}1.52(1.22, \\
1.85)\end{array}$ & $\begin{array}{l}1.33(1.12, \\
1.59)\end{array}$ & $\begin{array}{l}1.32(1.14, \\
1.54)\end{array}$ & $\begin{array}{l}1.30(1.09 \\
1.54)\end{array}$ & $\begin{array}{l}1.28(1.15 \\
1.43)\end{array}$ & $\begin{array}{l}1.30(1.11, \\
1.54)\end{array}$ \\
\hline Physical dating violence & $\begin{array}{l}2.17(1.22, \\
3.85)\end{array}$ & $\begin{array}{l}1.64(1.16, \\
2.27)\end{array}$ & $\begin{array}{l}1.67(1.14, \\
2.50)\end{array}$ & $\begin{array}{l}1.85(1.41, \\
2.44)\end{array}$ & $\begin{array}{l}1.72(1.23 \\
2.38)\end{array}$ & $\begin{array}{l}1.85(1.33 \\
2.56)\end{array}$ & $\begin{array}{l}1.79(1.25 \\
2.56)\end{array}$ & $\begin{array}{l}1.96(1.35, \\
2.78)\end{array}$ \\
\hline $\begin{array}{l}\text { Bullied on school } \\
\text { property }\end{array}$ & $\begin{array}{l}1.67 \text { (1.37, } \\
2.04)\end{array}$ & $\begin{array}{l}1.56(1.30, \\
1.85)\end{array}$ & $\begin{array}{l}1.43(1.23, \\
1.64)\end{array}$ & $\begin{array}{l}1.23(1.10 \\
1.39)\end{array}$ & $\begin{array}{l}1.35(1.20 \\
1.52)\end{array}$ & $\begin{array}{l}1.35(1.20 \\
1.54)\end{array}$ & $\begin{array}{l}1.25(1.14 \\
1.37)\end{array}$ & $\begin{array}{l}1.06(1.00, \\
1.12)\end{array}$ \\
\hline \multicolumn{9}{|l|}{ Substance use } \\
\hline Current smoking & $\begin{array}{l}2.38(1.67, \\
3.45)\end{array}$ & $\begin{array}{l}1.08(0.93 \\
1.22)\end{array}$ & $\begin{array}{l}1.75(1.37 \\
2.27)\end{array}$ & $\begin{array}{l}1.08(0.98 \\
1.19)\end{array}$ & $\begin{array}{l}1.54(1.27, \\
1.89)\end{array}$ & $\begin{array}{l}1.05(0.97 \\
1.15)\end{array}$ & $\begin{array}{l}1.67(1.30 \\
2.17)\end{array}$ & $\begin{array}{l}1.15(1.06, \\
1.25)\end{array}$ \\
\hline Current alcohol use & $\begin{array}{l}1.59(1.27 \\
2.00)\end{array}$ & $\begin{array}{l}1.14(0.99, \\
1.30)\end{array}$ & $\begin{array}{l}1.30(1.12, \\
1.49)\end{array}$ & $\begin{array}{l}1.09(0.99 \\
1.19)\end{array}$ & $\begin{array}{l}1.28(1.11, \\
1.47)\end{array}$ & $\begin{array}{l}1.11(1.01, \\
1.22)\end{array}$ & $\begin{array}{l}1.25(1.09 \\
1.43)\end{array}$ & $\begin{array}{l}1.10(1.02, \\
1.19)\end{array}$ \\
\hline Current marijuana use & $\begin{array}{l}1.47(1.20 \\
1.82)\end{array}$ & $\begin{array}{l}1.05(0.93 \\
1.18)\end{array}$ & $\begin{array}{l}1.25(1.11, \\
1.43)\end{array}$ & $\begin{array}{l}1.09(1.00, \\
1.18)\end{array}$ & $\begin{array}{l}1.19(1.08, \\
1.32)\end{array}$ & $\begin{array}{l}1.01(0.94, \\
1.09)\end{array}$ & $\begin{array}{l}1.14(1.03 \\
1.25)\end{array}$ & $\begin{array}{l}1.04(0.98 \\
1.10)\end{array}$ \\
\hline Ever cocaine use & $\begin{array}{l}0.92(0.51 \\
1.64)\end{array}$ & $\begin{array}{l}1.39(1.02, \\
1.89)\end{array}$ & $\begin{array}{l}0.80(0.62, \\
1.47)\end{array}$ & $\begin{array}{l}1.19(0.96 \\
1.47)\end{array}$ & $\begin{array}{l}1.01(0.62, \\
1.61)\end{array}$ & $\begin{array}{l}1.27(0.97, \\
1.64)\end{array}$ & $\begin{array}{l}1.67(0.72, \\
3.85)\end{array}$ & $\begin{array}{l}1.75(1.23, \\
2.50)\end{array}$ \\
\hline Ever heroin use & $\begin{array}{l}1.06(0.52 \\
2.17)\end{array}$ & $\begin{array}{l}2.27 \text { (1.37, } \\
3.85)\end{array}$ & $\begin{array}{l}1.22(0.68, \\
2.17)\end{array}$ & $\begin{array}{l}1.30(1.01, \\
1.67)\end{array}$ & $\begin{array}{l}1.61(0.76 \\
3.45)\end{array}$ & $\begin{array}{l}1.82(1.22, \\
2.70)\end{array}$ & $\begin{array}{l}1.79(0.72 \\
4.35)\end{array}$ & $\begin{array}{l}2.78(1.47, \\
5.26)\end{array}$ \\
\hline $\begin{array}{l}\text { Ever methamphetamine } \\
\text { use }\end{array}$ & $\begin{array}{l}0.83(0.52 \\
1.32)\end{array}$ & $\begin{array}{l}1.69(1.12, \\
2.50)\end{array}$ & $\begin{array}{l}1.28(0.67 \\
2.44)\end{array}$ & $\begin{array}{l}1.19(0.95 \\
1.52)\end{array}$ & $\begin{array}{l}1.49(0.78 \\
2.86)\end{array}$ & $\begin{array}{l}1.56(1.16, \\
2.13)\end{array}$ & $\begin{array}{l}2.13(0.70 \\
6.67)\end{array}$ & $\begin{array}{l}2.04(1.47, \\
2.78)\end{array}$ \\
\hline \multicolumn{9}{|l|}{ Sexual behavior } \\
\hline Currently sexually active & $\begin{array}{l}1.25(1.02, \\
1.52)\end{array}$ & $\begin{array}{l}1.01(0.89 \\
1.14)\end{array}$ & $\begin{array}{l}1.27(1.10, \\
1.47)\end{array}$ & $\begin{array}{l}1.10(1.01, \\
1.20)\end{array}$ & $\begin{array}{l}1.20(1.06, \\
1.35)\end{array}$ & $\begin{array}{l}1.04(0.96 \\
1.14)\end{array}$ & $\begin{array}{l}1.20(1.08, \\
1.33)\end{array}$ & $\begin{array}{l}1.10(1.01, \\
1.19)\end{array}$ \\
\hline $\begin{array}{l}\text { Sex with } 4 \text { or more in } \\
\text { life }\end{array}$ & $\begin{array}{l}1.89(1.00, \\
3.45)\end{array}$ & $\begin{array}{l}1.20(0.95 \\
1.54)\end{array}$ & $\begin{array}{l}1.15(0.85 \\
1.54)\end{array}$ & $\begin{array}{l}1.12(0.93 \\
1.35)\end{array}$ & $\begin{array}{l}1.04(0.82 \\
1.32)\end{array}$ & $\begin{array}{l}1.05(0.92 \\
1.20)\end{array}$ & $\begin{array}{l}1.20(0.95 \\
1.54)\end{array}$ & $\begin{array}{l}1.18(1.03 \\
1.35)\end{array}$ \\
\hline \multicolumn{9}{|l|}{ Early risk-taking behavior } \\
\hline Smoking under age 13 & $\begin{array}{l}1.20(1.01 \\
1.43)\end{array}$ & $\begin{array}{l}1.04(0.93 \\
1.18)\end{array}$ & $\begin{array}{l}1.23(1.04 \\
1.47)\end{array}$ & $\begin{array}{l}1.09(1.00 \\
1.19)\end{array}$ & $\begin{array}{l}1.22(1.05 \\
1.43)\end{array}$ & $\begin{array}{l}1.12(1.03 \\
1.23)\end{array}$ & $\begin{array}{l}1.10(0.98 \\
1.23)\end{array}$ & $\begin{array}{l}1.04(0.97 \\
1.12)\end{array}$ \\
\hline Drinking under age 13 & $\begin{array}{l}1.33(1.05 \\
1.69)\end{array}$ & $\begin{array}{l}1.08(0.93 \\
1.23)\end{array}$ & $\begin{array}{l}1.23(1.08, \\
1.41)\end{array}$ & $\begin{array}{l}1.11(1.00 \\
1.23)\end{array}$ & $\begin{array}{l}1.27(1.10, \\
1.45)\end{array}$ & $\begin{array}{l}1.14(1.03 \\
1.25)\end{array}$ & $\begin{array}{l}1.11(1.00, \\
1.22)\end{array}$ & $\begin{array}{l}1.10(1.02, \\
1.18)\end{array}$ \\
\hline $\begin{array}{l}\text { Marijuana use under age } \\
13\end{array}$ & $\begin{array}{l}1.14(0.87 \\
1.49)\end{array}$ & $\begin{array}{l}1.10(0.96 \\
1.27)\end{array}$ & $\begin{array}{l}1.19(0.98, \\
1.45)\end{array}$ & $\begin{array}{l}1.02(0.93 \\
1.12)\end{array}$ & $\begin{array}{l}1.16(0.98 \\
1.37)\end{array}$ & $\begin{array}{l}1.01(0.91 \\
1.11)\end{array}$ & $\begin{array}{l}1.27(1.08 \\
1.52)\end{array}$ & $\begin{array}{l}1.09(1.00 \\
1.19)\end{array}$ \\
\hline Sex under age 13 & $\begin{array}{l}1.39(0.80 \\
2.38)\end{array}$ & $\begin{array}{l}1.06(0.84 \\
1.33)\end{array}$ & $\begin{array}{l}1.20(0.84 \\
1.72)\end{array}$ & $\begin{array}{l}1.02(0.88 \\
1.19)\end{array}$ & $\begin{array}{l}1.09(0.81 \\
1.43)\end{array}$ & $\begin{array}{l}0.97(0.85 \\
1.09)\end{array}$ & $\begin{array}{l}1.02(0.84 \\
1.23)\end{array}$ & $\begin{array}{l}1.05 \\
1.22)\end{array}$ \\
\hline
\end{tabular}

Note. $R R$ relative risk, $C l=$ confidence interval. The numbers in bold indicate significance at $p<0.05$

\section{Early risk-taking behaviors associated with depressed mood and suicidal behaviors}

Smoking before the age of 13 was positively associated with suicide plan for both female and male adolescents. Drinking alcohol before the age of 13 was positively associated with suicide plan $(\mathrm{ARR}=1.14,95 \% \mathrm{CI}=1.02-$ $1.27, p<0.05)$ and suicide attempt $(\mathrm{ARR}=1.11,95 \% \mathrm{CI}=$ $1.03-1.20, p<0.01)$ for male adolescents.

\section{Discussion}

The prevalence of depressed mood and suicidal behaviors among CNMI high school adolescents was higher than their national prevalence in the US. Although male adolescents have a higher suicide rate in the US Trust Territories of the Pacific Islands [15], female adolescents are more likely to experience depressed mood and suicidal behaviors. The current study extends the finding 
that adolescents experiencing depressed mood and suicidal behaviors often engage in health risk behaviors. Specifically, use of "soft drugs" such as alcohol was associated with depressed mood, suicide ideation, suicide plan, and suicide attempt among female adolescents, whereas use of "hard drugs" such as cocaine showed greater associations among male adolescents.

In the 2017 CNMI YRBS, 40.7\% of adolescents reported being depressed, $25.0 \%$ reported suicide ideation, $22.8 \%$ reported formulating a suicide plan, and $13.6 \%$ attempted suicide. By contrast, in the 2017 national YRBS [22], 31.5\% reported being depressed, $17.2 \%$ reported suicide ideation, $13.6 \%$ reported formulating a suicide plan, and $7.4 \%$ attempted suicide. Thus, CNMI adolescents were more likely to experience depressed mood and suicidal behaviors than adolescents in the US mainland. Because more than half of the adolescents in this study were Pacific Islanders, including the indigenous population of the CNMI, distinct cultural factors may have affected these results [20]. Indigenous people commonly experience social and economic marginalization and consequent disparities in health [26]. In the US Census, CNMI had a poverty rate of $55.7 \%$ [27], while the national poverty rate was $12.3 \%$ [28]. These data suggest that economic barriers might prevent adolescents in the CNMI from accessing and receiving proper mental health care, such as counseling and therapy. Further research is also necessary to investigate the fear and stigmatization surrounding mental health in the Pacific Islands, as these may have acted as social barriers to adolescents consulting mental health professionals [29].

In this study, female adolescents were more likely to experience depressed mood, suicide ideation, suicide plan, and suicide attempt than male adolescents in the CNMI, perhaps because of transitioning gender roles. Traditionally, Micronesian societies were matrilineal, granting women important roles in the community and equal power as men. However, due to societal transition, the culture is shifting to patrilineality, increasing the economic and social marginalization of women [16]. One study implicated the low status of women as an important indicator of suicide in the Pacific Islands [13]. These societal transitions can cause female adolescents to be more vulnerable in society, which can possibly lead to depressed mood and suicidal behaviors.

Being in a physical fight and forced sexual intercourse were associated with depressed mood, suicide ideation, suicide plan, and suicide attempt. In Micronesian culture, including the CNMI, one of the major precipitating factors of suicide is conflict between the victim and someone very close to the victim [14]. Moreover, one study identified the dominant emotion at play in suicide in Micronesia as anger [15]. This suggests that adolescents who engage in conflict with another individual might be more likely to experience depressed mood and suicidal behaviors. Carrying a weapon was associated with suicide ideation for male adolescents and not for female adolescents. This finding is consistent with those indicating that male adolescents who carry a weapon and engage in physical fights in school and community settings are at increased risk for suicidal behaviors [30]. However, the variable that measures weapon carrying among adolescents does not determine whether the individual carried a weapon as a tool to cause violence or for self-protection as a result of feeling unsafe at school.

In this study, both female and male adolescents who experienced dating violence were more likely to experience suicide ideation, suicide plan, and suicide attempt. Studies have considered the effects of dating violence to occur exclusively among females on the assumption that female adolescents are more likely to experience severe forms of dating violence including sexual assault and physical injuries [31]. However, another study indicated that dating violence among adolescent relationships often involves the reciprocal use of violence by both partners [32]. Therefore, the effect of dating violence on depressed mood and suicidal behaviors should be further explored following a gender-inclusive approach, as both female and male adolescents might experience dating violence differently, and thus, it is crucial to consider their respective experiences [33]. Moreover, a study conducted in the US indicated that limited experience with romantic relationships causes most adolescents to have difficulty in dealing with and resolving problems they encounter in a relationship [34]. As a result, some may respond to relationship difficulties with violence while others may cope with self-inflicted violence, which can include suicidal behaviors.

In this study, there were significant gender differences in substance use behaviors associated with depressed mood, suicide ideation, suicide plan, and suicide attempt. Specifically, female adolescents reported more substance use behaviors involving "soft drugs" such as smoking, alcohol, and marijuana that were associated with depressed mood, suicide ideation, suicide plan, and suicide attempt. Male adolescents had more substance use behaviors involving "hard drugs" such as cocaine, heroin, and methamphetamine that were associated with depressed mood, suicide plan, and suicide attempt. The contrasting results for female and male adolescents' associations between substance use behaviors, depressed mood, and suicidal behaviors might have been due to gender differences in the motivation for substance use [35]. One study investigating motivations for alcohol use among adolescents showed conformity to be more strongly related to alcohol use among male adolescents, whereas coping is more strongly related to alcohol use in female adolescents [36]. This could suggest that male 
adolescents are more likely to engage in "soft drugs" to socially connect with their peers, while female adolescents do so to avoid and cope with problems that may be associated with depressed mood and suicidal behaviors.

\section{Limitations}

The present study is subject to several limitations. First, the questions related to mental health are limited. Questions on anxiety, post-traumatic stress disorder, or other past episodes related to mental health should be included to identify further psychological risk factors for depression and suicidal behaviors. Moreover, this study could not identify the impulsivity of suicidal behaviors among adolescents. A recent study suggested that a considerable number of suicide attempts occur due to sudden desires and were more common among younger people [37]. Second, all questions were self-reported, and the extent of under- or overreporting due to social desirability bias cannot be determined, although the survey questions demonstrated good test-retest reliability $[22,23]$. Because the current study did not include adolescents who were absent from school on the day of the study and those whose parents did not provide permission for participation, the possibility of selection bias may be considerable. Due to this selection bias, adolescents who were at a higher risk of depressed mood, suicidal behaviors, and health risk behaviors may have been excluded from the study. Third, the current study followed a cross-sectional design and was unable to determine the causal relationship between health risk behaviors, depressed mood, and suicidal behaviors. The study also lacked a control group, and therefore could not determine other possible factors that might have affected the results. Fourth, the current study examined a number of combinations of independent and dependent variables, which might have yielded spurious statistical significance due to multiple comparisons. To counter this issue, we focused on our findings of associations of health risk behaviors that were consistently statistically significant across depressed mood, suicide ideation, suicide plan, and suicide attempt. Finally, survey questions related to the sociodemographic characteristics of adolescents were limited. During adolescence, social, environmental, situational, and cultural factors can significantly affect well-being $[7,10]$, and therefore, there could have been important confounders associated with depressed mood, suicidal behaviors, and health risk behaviors that were missed. Thus, residual confounding by variables missing from the questionnaire might have affected the associations found in the current study. Questions related to adolescents' socioeconomic status, family structure, and peer involvement should be included to further investigate the factors associated with depressed mood and suicidal behaviors.

\section{Conclusion and public health implications}

The prevalence of depressed mood and suicidal behaviors among high school adolescents was found to be higher in the CNMI than in the US mainland. Mental health among the indigenous population of the US Pacific territories should be further investigated to strengthen the monitoring of suicide and to enhance the quality of mental health data from the culturally distinct territories of the US. Moreover, when implementing public health interventions with limited resources, school health professionals might consider implementing and evaluating intervention programs for high school adolescents that concurrently address mental health issues such as depressed mood, suicide, and health risk behaviors. Specifically, the prevention of substance use, dating violence, bullying, and related topics could be mentioned while addressing depression and suicide. Furthermore, this study recommends implementing risk behavior and suicide prevention interventions separately for female and male adolescents, as they experience certain health risk behaviors, depressed mood, and suicidal behaviors differently.

\section{Abbreviations}

ARR: Adjusted relative risk; CDC: Centers for Disease Control and Prevention; $\mathrm{Cl}$ : Confidence interval; CNMI: Commonwealth of the Northern Mariana Islands; YRBS: Youth Risk Behavior Survey

\section{Acknowledgments \\ Not applicable.}

\section{Author contributions}

All authors contributed to the study design and management. JLS and AS analyzed and interpreted the data. JLS wrote the manuscript. JLS, AS, and MJ all revised, edited, and approved the manuscript.

Funding

None.

Availability of data and materials

The data that support the findings of this study are available from the CDC upon request on their website. https://www.cdc.gov/healthyyouth/data/yrbs/ contact.htm

Ethics approval and consent to participate

This study was exempt from the Research Ethics Committee of the Graduate School of Medicine, The University of Tokyo, Japan, as the data set did not include any personally identifiable information. Prior to this study, the CDC approved the protocol for all YRBS conducted in the US. The survey was designed to protect adolescents' privacy through anonymity and encourage voluntary participation. Before administering the survey, local parental permission procedures were followed. Data were obtained from the CDC upon request.

\section{Consent for publication}

Not applicable.

Competing interests

The authors declare that they have no competing interests. 
Received: 10 September 2019 Accepted: 6 April 2020

Published online: 15 April 2020

\section{References}

1. Centers for Disease Control and Prevention. National suicide statistics: Ten leading causes of death by age group. 2017. Available at: https://www.cdc gov/injury/wisqars/pdf/leading_causes_of_death_by_age_group_2017-508. pdf. Accessed 8 July 2019.

2. Centers for Disease Control and Prevention. Violence Prevention. Preventing Suicide. 2017. Available at: https://www.cdc.gov/violenceprevention/suicide/ fastfact.html. Accessed 8 July 2019.

3. Paxton RJ, Valois RF, Watkins KW, Huebner ES, Drane JW. Associations between depressed mood and clusters of health risk behaviors. Am J Health Behav. 2007;31(3):272-83.

4. Richardson AS, Bergen HA, Martin G, Roeger L, Allison S. Perceived academic performance as an indicator of risk of attempted suicide in young adolescents. Arch Suicide Res. 2005;9(2):163-76.

5. Jiang Y, Perry DK, Hesser JE. Adolescent suicide and health risk behaviors Rhode Island's 2007 youth risk behavior survey. Am J Prev Med. 2007;38: 551-5.

6. Nock MK, Borges G, Bromet E, Cha CB, Kessler RC, Lee S. Suicide and suicidal behavior. Epidemiol Rev. 2008;30(1):133-54.

7. Cha CB, Franz PJ, Guzman EM, Glenn CR, Kleiman EM, Nock MK. Annual research review: suicide among youth-epidemiology, (potential) etiology, and treatment. J Child Psychol Psychiatry. 2018;59(4):460-82.

8. Chatterji P, Dave D, Kaestner R, Markowitz S. Alcohol abuse and suicide attempts among youth. Econ Hum Biol. 2004;2(2):159-80.

9. Hallfors DD, Waller MW, Ford CA, Halpern CT, Brodish PH, Iritani B. Adolescent depression and suicide risk: association with sex and drug behavior. Am J Prev Med. 2004;27(3):224-31.

10. Shilubane HN, Ruiter RA, van den Borne B, Sewpaul R, James S, Reddy PS. Suicide and related health risk behaviours among school learners in South Africa: results from the 2002 and 2008 national youth risk behaviour surveys. BMC Public Health. 2013;13:926

11. Milner A, De Leo D. Suicide research and prevention in developing countries in Asia and the Pacific. Bull World Health Organ. 2010;88(10):795-6.

12. Guampedia. Suicide in Micronesia. 2018. Available at: https://www.guampedia. com/contemporary-guam-suicide-in-micronesia/. Accessed 20 July 2019.

13. Booth H. Pacific Island suicide in comparative perspective. J Biosoc Sci. 1999;31(4):433-48

14. Rubinstein D. Youth Suicide and Social Change in Micronesia. Occasional Papers (Kagoshima Research Center for the Pacific Islands). 2002;36:33-41.

15. Ran MS. Suicide in Micronesia: a systematic review. Prim Psychiatry. 2007; 14(11):80-7.

16. Hezel F. Suicide and the Micronesian family. Contemp Pac. 1989;1(1):43-74.

17. Booth $\mathrm{H}$. The evolution of epidemic suicide on Guam: context and contagion. Suicide Life Threat Behav. 2010;40(1):1-13.

18. Jiang Y, Perry DK, Hesser JE. Suicide patterns and association with predictors among Rhode Island public high school students: a latent class analysis. Am J Pub Health. 2010;100:9.

19. Centers for Disease Control and Prevention. 2017 YRBS User's Guide 2018. Available at: https://www.cdc.gov/healthyyouth/data/yrbs/data.htm. Accessed 2 Sept 2018.

20. Else IRN, Andrade NN, Nahulu LB. Suicide and suicidal-related behaviors among indigenous Pacific islanders in the United States. Death Stud. 2007; 31:479-501.

21. Brener ND, Kann L, McManus T, Kinchen SA, Sundberg EC, Ross JG. Reliability of the 1999 youth risk behavior survey questionnaire. J Adolesc Health. 2002;31(4):336-42.

22. Kann L, McManus T, Harris WA, et al. Youth risk behavior surveillance United States, 2017. MMWR Surveill Summ. 2018;67(8):1-114.

23. May A, Klonsky ED. Validity of suicidality items from the youth risk behavior survey in a high school sample. Assessment. 2011;18(3):379-81.

24. Veazie PJ. When to combine hypotheses and adjust for multiple tests. Health Serv Res. 2006:41(3):804-18.

25. Streiner DL. Best (but oft-forgotten) practices: the multiple problems of multiplicity-whether and how to correct for many statistical tests. Am J Clin Nutr. 2015;102(4):721-8.

26. Pollock NJ, Naicker K, Loro A, Mulay S, Colman I. Global incidence of suicide among indigenous peoples: a systematic review. BMC Med. 2018;16(1):145.
27. CNMI Department of Commerce. HIEC 2016 Population. 2016. Available at: http://commerce.gov.mp/hies-2016-population. Accessed July 8, 2019.

28. United States Census Bureau. Income, poverty, and health insurance coverage in the United States: 2017. 2018. Available at: https://www.census. gov/content/dam/Census/library/publications/2018/demo/p60-263.pdf. Accessed 2 Sept 2019.

29. Subica AM, Aitaoto N, Sullivan JG, Henwood BF, Yamada AM, Link BG. Mental illness stigma among Pacific islanders. Psychiatry Res. 2019;273:578-85.

30. Nickerson A, Slater ED. School and community violence and victimization as predictors of adolescent suicidal behavior. School Psych Rev. 2009;38(2): 218-32.

31. Banyard VL, Cross C. Consequences of teen dating violence: understanding intervening variables in ecological context. Violence Against Women. 2008; 14(9):998-1013.

32. Taquette SR, Monteiro DLM. Causes and consequences of adolescent dating violence: a systematic review. J Inj Violence Res. 2019;11(2):137-47.

33. Hamel J. Gender-inclusive treatment of intimate partner abuse: evidencebased approaches. 2nd ed. New York, US: Springer Publishing Co.; 2014.

34. Shulman S, Connolly J. The challenge of romantic relationships in emerging adulthood: reconceptualization of the field. Emerging Adulthood. 2013;1(1): 27-39.

35. Kuntsche E, Wicki M, Windlin B, Roberts C, Gabhainn SN, van der Sluijs W, et al. Drinking motives mediate cultural differences but not gender differences in adolescent alcohol use. J Adolesc Health. 2015;56(3):323-9.

36. Buckner JD, Shah SM. Fitting in and feeling fine: conformity and coping motives differentially mediate the relationship between social anxiety and drinking problems for men and women. Addict Res Theory. 2015;23(3):231-7.

37. Rimkeviciene J, O'Gorman J, De Leo D. Impulsive suicide attempts: a systematic literature review of definitions, characteristics and risk factors. J Affect Disord. 2015;171:93-104.

\section{Publisher's Note}

Springer Nature remains neutral with regard to jurisdictional claims in published maps and institutional affiliations.

Ready to submit your research? Choose BMC and benefit from:

- fast, convenient online submission

- thorough peer review by experienced researchers in your field

- rapid publication on acceptance

- support for research data, including large and complex data types

- gold Open Access which fosters wider collaboration and increased citations

- maximum visibility for your research: over $100 \mathrm{M}$ website views per year

At $\mathrm{BMC}$, research is always in progress.

Learn more biomedcentral.com/submissions 